\title{
HIGH-PRECISION BEAM-BASED RF PHASE STABILIZATION AT JEFFERSON LAB*
}

\author{
V. A. Lebedev, J. Musson, and M. G. Tiefenback ${ }^{\dagger}$, \\ Thomas Jefferson National Accelerator Facility, Newport News, VA
}

\begin{abstract}
A non-invasive monitor has been implemented for the relative phase of the beam and the accelerating RF of each of the two CW superconducting linacs at TJNAF. Its noise level and resolution are below $0.1^{\circ}$ at beam currents as low as 2 microamperes, and it been successfully tested for use in feedback correction for the overall RF accelerating phase of each linac. The $70 \mathrm{MHz}$ frequency reference in each linac is phase modulated by approximately $\pm 0.05^{\circ}$ at different frequencies ( 383 and $397 \mathrm{~Hz}$ ). A single analog beam position signal from a dispersive region is used to monitor the resulting micron-level position modulation via off-theshelf lock-in amplifiers, which also supply the modulation signals. The off-crest phase is proportional to the first harmonic coefficient of the energy modulation. This technique can be applied as well to pulsed machines and to systems for which the beam-to-RF relative phase may be non-zero. Other applications are discussed.
\end{abstract}

\section{INTRODUCTION}

CEBAF is a superconducting $\mathrm{CW}$ recirculating electron accelerator, one design goal of which was to achieve the very low RMS energy spread of $2.5 \times 10^{-5}$. Achieving this requires both a short electron bunch and an RF accelerating system with low phase noise. Past and future Master Oscillator (MO) system sources meet the requirements[1], but phase drifts in the MO distribution have required attention since early commissioning activities. Improved thermal regulation of the distribution lines has reduced the intrinsic drifts to within $\pm 2^{\circ}$, and software feedback loops and manual readjustments have provided adequate phase control to date. However, the continuous phase monitoring provided by this system will help in maintaining the design energy spread goal by providing the information needed for immediate correction of the residual drifts.

\section{PHASE SENSITIVITY}

Changes in beam energy are monitored through the beam position monitor (BPM) system in dispersive regions, and a feedback system for energy correction has recently been provided[2] at the experimental hall transport lines (point of delivery monitoring and correction). Stabilizing beam energy at one point in the system, however, does not stabilize the energy spread, and under certain conditions may

\footnotetext{
* Work supported by the U.S. Department of Energy under contract number DE-AC05-84ER40150.

† Email: tiefen@jlab.org
}

not sufficiently stabilize the beam energy for other acceleration passes. The energy spread depends upon many factors, including the bunch length, the overall coherence of the linac accelerating systems (periodically adjusted to correct for local hardware drifts), and the overall vector phase of the linac with respect to the various recirculating beams.

This system is intended to improve monitoring and correction of the overall phase of the linac. We have adapted the signal processing hardware used in the Hall B nanoampere BPM system[3] to measure the global phase of each linac with respect to the beam with better than $0.1^{\circ}$ resolution. The technique involves phase modulation of each linac at the $0.05^{\circ}$ level, contributing at the $3 \times 10^{-6}$ level to the overall RMS energy spread.

\subsection{Induced Energy Spread}

The energy gain from an RF system, initially at some phase $\psi$ with respect to crest, by a perturbation $\delta$ at an angular frequency $\omega$, is of the form

$$
\cos (\psi+\delta \sin (\omega t))
$$

In addition to a slight level shift, there are first and second harmonic contributions to the energy of the beam approximately given by

$$
\delta \sin (\psi) \sin (\omega t)+\frac{\delta^{2}}{4} \cos (\psi) \cos (2 \omega t) .
$$

The $\sin (\omega t)$ term provides the detection capability used here. It is possible that the $\cos (2 \omega t)$ term may be usable as a "system on and working" flag. In the CEBAF system, each linac provides about half of the beam energy. For a modulation amplitude of $\pm 0.05^{\circ}$ at an off-crest phase of $0.5^{\circ}$, these two terms provide RMS additions to the energy spread of $2.5 \times 10^{-6}$ at the fundamental harmonic and $1.3 \times$ $10^{-7}$ at the second harmonic.

\section{SYSTEM LAYOUT}

The detection system is presently installed in the "spreader" (region for vertical separation of the multiple beam passes) at the end of the South Linac (see Fig. 1. The dispersion at the BPM used for detection is $1.4 \mathrm{~m}$, so that a $2.5 \times 10^{-6}$ RMS energy (momentum) modulation results in an RMS beam position modulation of 3.5 microns. This location was chosen because a single BPM here allows monitoring of both linacs for all beam delivery configurations. Adding BPM pick-ups for recirculated beam is an option discussed below. 


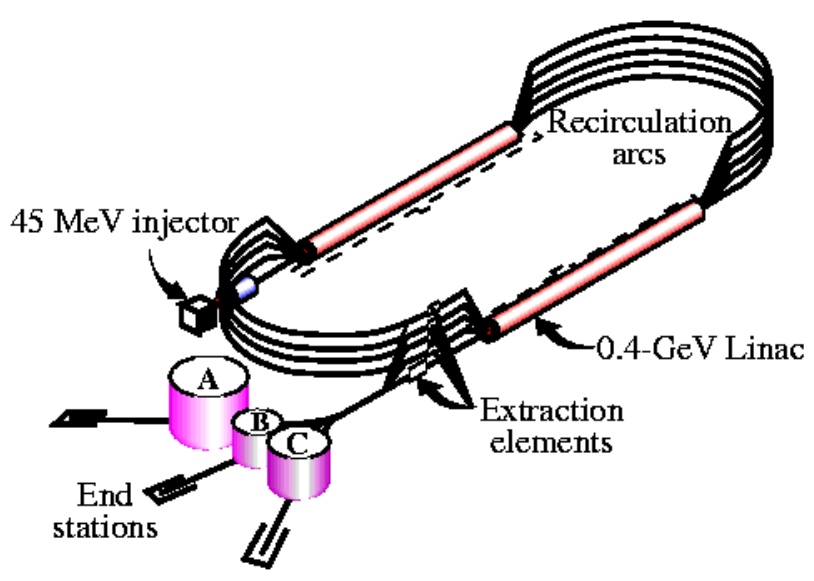

Figure 1: Physical layout of CEBAF beam lines. The BPM used for signal detection is near the foreground end of the right-hand linac.

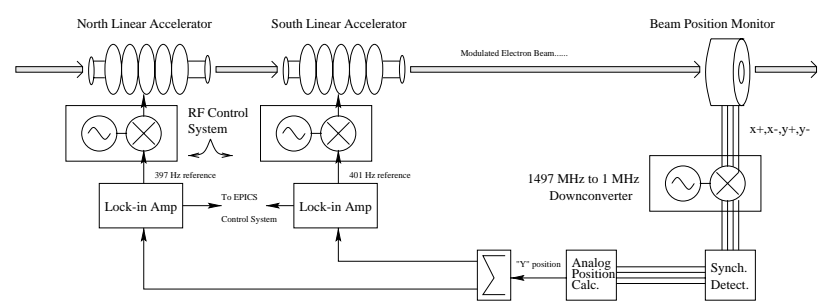

Figure 2: Layout of Master Oscillator Modulation system at CEBAF. Each linac uses one lock-in amplifier for a phase modulation reference and to detect the position modulation resulting from the energy modulation. A single BPM serves to detect the beam position for both systems.

\subsection{Phase Modulation Subsystem}

The overall MO Modulation system is shown in Fig. 2. It consists of commercial, off-the-shelf, lock-in amplifiers (model 7220 from EG\&G), a phase modulation system, and a beam position monitor (BPM) pick-up. The lock-in units both provide the phase modulation source and detect the resulting beam energy modulation,

In each linac, the RF systems use $1427 \mathrm{MHz}$ and $70 \mathrm{MHz}$ signals supplied by the MO system to control the cavities at $1497 \mathrm{MHz}$. The $70 \mathrm{MHz}$ signal is phase modulated at different frequencies in each linac. The modulation frequencies are $383 \mathrm{~Hz}$ for the North (first) Linac and $397 \mathrm{~Hz}$ for the South Linac. These frequencies $( \pm 7 \mathrm{~Hz}$ from the 13th harmonic of $30 \mathrm{~Hz}$ ) were chosen for their low background noise and for isolation from the experimentally sensitive 30 $\mathrm{Hz}$ frequency at which the sign of beam spin polarization is changed. The phase modulators were carefully adjusted to give zero amplitude modulation of the $70 \mathrm{MHz}$ signal at the fundamental phase modulation frequency. Any such amplitude modulaton would couple directly into gradient modulation of each linac, interfering with the phase detection.

\subsection{Sensor and Calibration}

The BPM used for detection is of the "4-channel" type, with independent amplifier and demodulation channels for each of the four pickups. Each of these four signals is continuously available (low-pass filtered to $50 \mathrm{kHz}$ ). The standard BPM system digitizes these signals at $60 \mathrm{~Hz}$, numerically processing them to provide laboratory frame vertical and horizontal beam positions. The pick-ups in the CEBAF accelerator are rotated $45^{\circ}$ about the beam axis away from horizontal and vertical to avoid photo-emission interference from synchrotron radiation. The analog signal can be used to detect very small position modulations by using an analog processing circuit to generate laboratory frame analog beam position signals. For convenience, the analog signal processor both provides lab frame outputs and normalizes the signal against the beam current, providing a sensitivity of $750 \mathrm{mV} / \mathrm{mm}$. The vertical position output is used by the two GPIB-interfaced lock-in amplifiers, the reference from each of which supplies the phase modulation reference for one linac.

The overall system sensitivity to changes in the linac RF phase was measured so that $1^{\circ}$ of indicated phase error would correspond to the same shift of the global phase control for each linac. The measured system sensitivities were $9000 \mu \mathrm{V} / \mathrm{deg}$ for the North Linac and $6430 \mu \mathrm{V} / \mathrm{deg}$ for the South Linac. This correlates well with the expected sensitivities. Sideband power levels measured on the 70 $\mathrm{MHz}$ signal of the linacs at the relevant phase modulation frequency at $-62 \mathrm{dBc}$ for the North Linac and $-67 \mathrm{dBc}$ for the South Linac, correspond to $0.07^{\circ}$ and $0.05^{\circ}$ of phase modulation. From the measured sensitivity of the analog bpm signal, the known beam line dispersion, and knowing that the energy gain of each linac is about $47 \%$ of the total beam energy for the first pass beam (in addition to the injector energy), the expected system sensitivity should be $7400 \mu \mathrm{V} / \mathrm{deg}$ for the North Linac and $5300 \mu \mathrm{V} / \mathrm{deg}$ for the South Linac. These values are listed in Table 1.

Table 1: System Sensitivity( $\mu \mathrm{V} / \mathrm{deg})$, estimated vs. measured

\begin{tabular}{|l|c|c|}
\hline Linac & Estimate & Measured \\
\hline North Linac & 7400 & 9000 \\
South Linac & 5300 & 6400 \\
\hline
\end{tabular}

\section{PERFORMANCE}

The system as presently configured can only be used with continuous beam, although this is an artificial limitation caused by the analog signal processing. The current normalization provided in the analog bpm processing circuit induces too much noise when the beam current is lower than about $2 \mu \mathrm{A}$. Although the zero current periods between beam macropulses during pulsed mode operation could be handled by gating the signals to the lock-ins, it 
might be better to extend the operating range to lower current by doing the current normalization after the lock-in signal extraction. This would have the added benefit of providing a useful signal for pulsed beam, although the integration period might have to be increased significantly to improve the signal-to-noise for this case.

A plot of the measured phase errors over a thirty minute period for the two linacs is shown in Fig. 3. The coherent portion of the signals in the two linacs is very possibly due to a modulation of the beam phase from the injector, but the roughly 20 minute oscillation of the South Linac phase with respect to that of the North Linac is probably due to residual thermal regulation effects in the MO distribution line for the South Linac. The noisy portion of the traces at the far left resulted from a short period of pulsed beam. Shifts of $0.1^{\circ}$ in the global phase set point for each linac can readily be detected when $\mathrm{CW}$ beam is present.

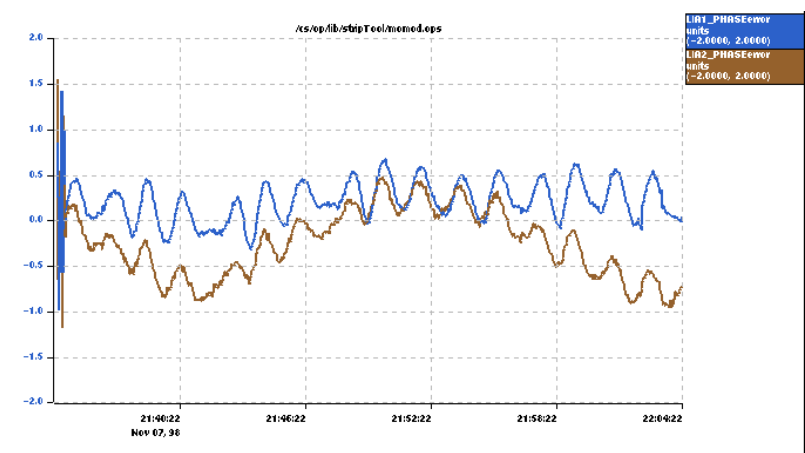

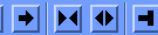

Figure 3: Thirty minute data stream from MO Modulation system for both linacs. The upper trace is the North Linac, and the lower trace is the South Linac. The vertical scale is $\pm 2^{\circ}$. See text for discussion.

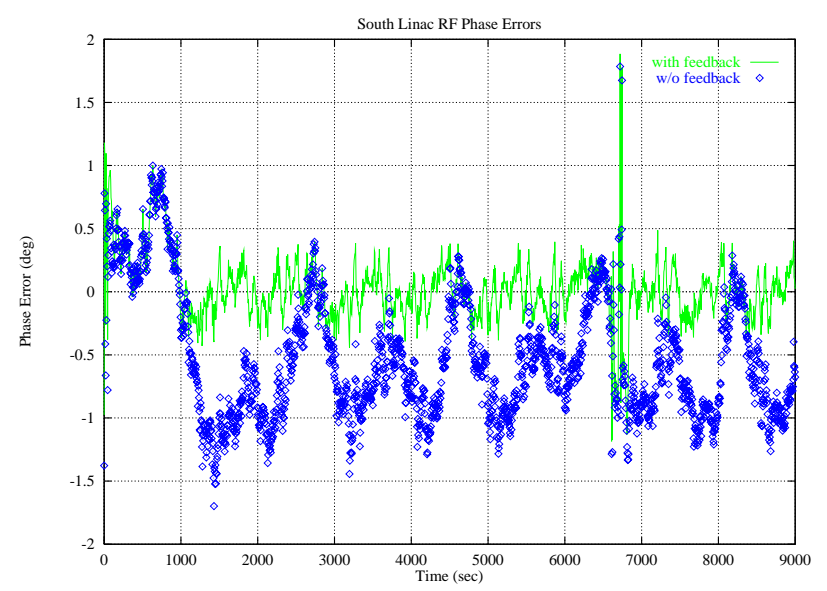

Figure 4: A $2.5 \mathrm{hr}$ data sample for the measured South Linac RF phase with feedback enabled. The upper trace is the actual measured phase error signal, while the lower trace has the feedback correction added back to recover the uncorrected phase drift. See the text for discussion.
The result of our first efforts at using this monitor as a feedback signal are shown in Fig. 4 for the South Linac. The phase correction is done in software by simple deadband limiting $\left( \pm 0.3^{\circ}\right)$, with corrections applied in discrete $0.1^{\circ}$ steps at intervals of one second. The data shown were taken at five-second intervals. The lower trace is the measured phase error offset by the cumulative shift of the linac phase setpoint, while the upper trace is the feedbacklimited actual phase error. The two traces are the same for the first 1000 seconds after beam is restored (near the lefthand side of the plot) until the feedback software is enabled.

The EG\&G 7220 amplifiers are typically configured to average over a 1 second period. Improvements in resolution are possible with longer integration periods, but not clearly beneficial in this application. The empirical noise floor for beam measurements at low beam currents is consistent with detection of RMS beam motion at the $0.5 \mu \mathrm{m}$ level.

\section{CONCLUSION AND FUTURE APPLICATIONS}

The present system monitors the relative phase of the beam and the RF in each linac for only the first acceleration pass. It provides data suitable for use by a feedback system which will maintain the RF of each linac on crest in each linac to within a few tenths of a degree. Similar detectors in the transport lines for the experimental halls would allow for point of delivery cresting of the multipass RF for each linac. Adding additional detector systems in the recirculation arcs (peak dispersion $2.5 \mathrm{~m}$ ) would allow for differential phase monitoring for recirculated beam. This would provide a sensitive monitor for the variations in the recirculation path length for $\mathrm{CW}$ beam, something we are presently able to measure only with short beam pulses. Such readily available digital information would be useful in automating the path length correction procedures.

Another application of interest is in monitoring the phase of one eight-cavity cryomodule in the injector. This section of the RF system is presently configured $10^{\circ}$ off-crest to provide a coherent energy spread along the electron bunch. The bunch is then magnetically compressed in the injection chicane as the beam from the injector is joined with the recirculating electrons before passing through the main linacs.

\section{REFERENCES}

[1] A Low-Cost, NIST-Traceable, High Performance Dielectric Resonant Master Oscillator for the Thomas Jefferson National Accelerator Facility, Larry Doolittle, et al., these proceedings

[2] Fast Digital Feedback System for Energy and Beam Position Stabilization, V. A. Lebedev, R. Dickson, these proceedings

[3] 1 nA Beam Position Monitoring System, Rok Ursic, et al., Proc. of the 1997 Part. Acc. Conf., Vancouver, B. C., Canada, 12-16 May, 1997. 